J. Clin. Chem. Clin. Biochem.

Vol. 18, 19.80, pp. 501-510

\title{
Der Einfluß von Meßzeit und Reaktionsmilieu bei kinetischen immunturbidimetrischen Proteinbestimmungen ${ }^{1}$ )
}

\author{
Von R. Müller-Matthesius und C. Opper
}

Aus den Forschungslaboratorien der Behringwerke AG, Marburg/Lahn

(Eingegangen am 23. November 1979/20. März 1980)

Zusammenfassung: Das Bezugssystem immunturbidimetrischer Bestimmungen, die Trübungskurve, hängt bei der kinetischen fixed-time-Technik von Meßbeginn, Meßzeitraum und Reaktionsmilieu ab. Am Beispiel des Systems IgG-anti-IgG wird gezeigt, wie die Meßbedingungen optimiert werden können. Besonders berücksichtigt werden hierbei einige der in größeren Laboratorien verwendeten Analysenautomaten (ABA-100, CentrifiChem, Cobas Bio, GEMSAEC, Gilford 3500).

\section{Influence of measurement time and reaction medium on kinetic immunturbidimetric protein determination}

Summary: For fixed-time rate analysis, the turbidity curve, which is the reference system of immunturbidimetric protein determination, depends on the time of first measurement, on the subsequent measurement time interval and on the composition of the reaction medium. The IgG/anti-IgG system has been chosen as an example to demonstrate how the reaction parameters can be optimized.

In this context particular consideration has been given to some of the analyzers commonly used in larger laboratories (ABA-100, CentrifiChem, Cobas Bio, GEMSAEC, Gilford 3500).

\section{Einleitung}

Seit 1974 wèrden für verschiedene Analysenautomaten kinetische Vàrianten der im Prinzip alten immunturbidimetrischen bzw. -nephelometrischen Plasmaproteinbestimmung publiziert (1-13).

In der Routine konkurriẹren gegenwärtig zwei kinetische Techniken. Bei der einen mißt man die Geschwindigkeitsmaxima ("peak rate") der Streulichtzunahme $(3,6)$. Da hierbei eine kontinuierliche Differenzierung des Meß. signals nach der Zeit durchgeführt werden muß, bleibt das Verfähren speziellen Geräten vorbehalten.

Die andere kinetische Technik, das sogenannte fixedtime-Verfahren, läßt sich besonders in Form der Trübungsmessung an vielen der bereits in der klinischen Routine vorhandenen Photometern und Automaten anwenden und besitžt daher a priori eine allgemeinere $\mathrm{Be}$ deutung.

1) Einige Ergebnisse der vorliegenden Arbeit waren bereits Teil eines Postervortrages auf der Jahrestagung der Deutschen und Osterreichischen Gesellschaften für Klinische Chemie, Salzburg, März 1979.
Ebenso wie bei den turbidimetrischen bzw. nephelometrischen Endwertverfahren findet man bei kinetischen Meßtechniken ähnliche Abhängigkeiten der Änderung des Meßsignals von der Antigenkonzentration wie bei den klassischen Präzipitationstechniken nach Heidelberger \& Kendall. Schultze \& Schwick (14) wiesen jedoch schon 1959 darauf hin, daß langsam aggregierende Antigen-Antikörper-Verbindungen bei kurzer Reaktionsdauer photometrisch nicht erfaßt werden und Trübungskurven dann schmaler als Präzipitationskurven nach Heidelberger ausfallen.

Aufgrund dieser Befunde muß man bei kinetischen fixed-time-Messungen Abhängigkeiten der Trübungskurve von Meßzeitraum und Reaktionsmilieu erwarten, die bisher relativ wenig untersucht wurden. So wurde die Herabsetzung der Reaktionsgeschwindigkeit durch Salze und verschiedene niedermolekulare organische Verbindungen $z$ war vielfach publiziert $(4,13,15-20)$, Konsequenzen für die praktische Durchführung von Antigenbestimmungen jedoch kaum gezogen.

In der vorliegenden Arbeit werden deshalb aus einer mehr pragmatischen Sicht Kriterien für die Wahl des 
Meßzeitraumes und für die Zusammensetzung des Reaktionsmediums am Beispiel der immunturbidimetrischen fixed-time-Bestimmung des IgG erörtert. Durch zahlreiche weitere Messungen konnte sichergestellt werden, daß sich bei anderen Proteinen prinzipiell ähnliche Verhältnisse ergeben.

\section{Material und Methoden}

\section{G eräte}

Manuelle Messungen: Analogphotometer $1101 \mathrm{M}$ der Firma Eppendorf Gerätebau, Hamburg, mit Küvettenwechselautomat 2705, CKE-Rechner 6453/Drucker 6522, Kompensationsschreiber 6511 .

Automaten: CentrifiChem 400 (Firma Union Carbide), Cobas Bio (Firma Hoffmann-La Roche), Gilford 3500, GEMSAEC (Firma Electro Nucleonics), ABA-100 (Firma Abbott).

\section{Reagenzien}

Standard: T Protein-Standard-Serum, Behringwerke AG, Bestell-Nr. $\emptyset$ SKT 04, IgG-Konzentration $10,87 \mathrm{~g} / \mathrm{l}$, bestimmt nach der Methode der einfachen radialen Immundiffusion mit Partigen ${ }^{\circledR}$-Platten der Behringwerke. Es handelt sich um ein stabilisiertes gepooltes Humanserum von gesunden Spendern.

Antiserum: T Antiserum gegen Human-IgG ( $\gamma$-Kette) vom Kaninchen, Behringwerke AG, Bestell-Nr. ØSBT 04.

Isotonische Natriumchlorid-Lösung: $9,0 \mathrm{~g} / 1 \mathrm{NaCl}$, azidfrei.

Phosphatgepufferte Natriumchlorid-Lösung: 8,62 $\mathrm{g} \mathrm{Na}_{2} \mathrm{HPO}_{4} \cdot$ $2 \mathrm{H}_{2} \mathrm{O}, 2,48 \mathrm{~g} \mathrm{KH}_{2} \mathrm{PO}_{4}$ und $8,5 \mathrm{~g} \mathrm{NaCl}$ in 11 Wasser.

Polyethylenglykol 6000: Serva, Heidelberg, Bestell-Nr. 33137.

Weitere Angaben zur Reagenzzusammensetzung siehe „Ergebnisse und Diskussion".

\section{Durchführung.und Messungen}

\section{Manuelle Untersuchungen}

$1000 \mu \mathrm{l}$ der antiserumhaltigen Reagenz-Lösung wurden in einer Halbmikro-Küvette vorgelegt, $20 \mu 1$ T Protein-Standard-Serum in unterschiedlicher Verdünnung (isoton. Natriumchlorid-Lösung) auf einem geeigneten Plastikspatel zugefügt und sofort gemischt. Bei laufendem Schreiber markierten die Spatelbewegungen den Reaktionszeitpunkt 0. Die Zunahme der Absorption wurde bei Raumtemperatur und der Wellenlänge $\mathrm{Hg} 334 \mathrm{~nm}$ mit der Papiervorschubgeschwindigkeit $2 \mathrm{~cm} / \mathrm{min}$ und der Spreizung $20 \mathrm{~cm}$ für $A=0-1$ registriert. Temperaturschwankungen von einigen Grad Celsius hatten keinerlei Einfluß auf das Ergebnis.

Bei kinetischen fixed-time-Messungen wurde die Absorption in bekannter Weise zu zwei Zeitpunkten $t_{1}$ und $t_{2}$ abgelesen und die Differenz $\Delta$ A gebildet (28).

Bei Messungen gegen einen in einer gesonderten Küvette angesetzten Reagenzien-Leerwert wurden die Küvetten vor Zugabe des Standard-Serums verglichen und Unterschiede rechnerisch korrigiert. Da unverdünntes Standard-Serum einen Absorptionsanstieg von nur 0,032 verursachte, konnten Proben-Leerwerte generell vernachlässigt werden.

Unspezifische Trübungen wurden durch Weglassen von Antiserum bzw. Standard-Serum kontrolliert und im positiven Falle (z. B. nach sehr langen Reaktionszeiten) rechnerisch korrigiert.

\section{Automaten}

Allgemeine Meßbedingungen

Temperatur: $+25^{\circ} \mathrm{C}$

Wellenlänge: $340 \mathrm{~nm}$ (ABA-100: $340 / 380 \mathrm{~nm}$ )

Testkonzentrationen: $9 \mathrm{~g} / 1$ Natriumchlorid, $10 \mathrm{~g} / 1$ Polyethylenglykol 6000, Antiserum 1:14,6.
Abweichungen von diesen Testkonzentrationen ergeben sich bei Tabelle 3 und sind dort gekennzeichnet.

Mit vorstehendem Reaktionsmedium wurde keine Optimierung quantitativer IgG-Bestimmungen angestrebt. Durch die Wahl eines gemeinsamen Reaktionsmilieus ist vielmehr beabsichtigt, individuelle Effekte der verschiedenen Geräte bei gleicher Reagenzzusammensetzung aufzuzeigen.

\section{CentrifiChem 400}

Pipettor: $5 \mu$ l Probe (Verdünnungsreihe des Standard-Serums), $60 \mu 1$ Probe + Verdünnungsmittel (bidest. Wasser), $250 \mu 1$ Reagenz.

Analyzer: $t_{0}$ time delay $5 \mathrm{~s}$, abnormal absorbance $1,0 \mathrm{U}$, blank AUTO, test mode Term, print out ABS, standard value 0000 testcode 00 .

Spezielle Bedingungen, Abbildung 9: die Testlösungen wurden eine Stunde vor Meßbeginn in Eppendorf-Gefäßen angesetzt und anschließend der Zentrifugiereffekt am CẹntrifiChem analysiert. Hierbei wurden die Differenzen zu den in I gespeicherten Absorptionen im Abstand von $1 \mathrm{~min} 45 \mathrm{~s}$ ( $\Delta t$ time interval) insgesamt neunmal (number of prints) ausgedruckt, ihre Absoluthöhe nach Ausdruck von Speicher I berechnet.

Spezielle Bedingungen, Abbildung 10: $\Delta t$ time interval $5 \mathrm{~min}$, number of prints 1 .

$A B A-100$

FRR-Technik (First Revolution Read) in Normalposition des Photometers, bei Analysis Time $10 \mathrm{~min}$. Dies entspricht einer 2-Punkt-fixed-time-Messung mit $t_{1}=10 \mathrm{~s}$ und $t_{2}=10 \mathrm{~min} 10 \mathrm{~s}$ (Bei einer Analysis Time von $5 \mathrm{~min}$ wird der erste Meßwert bereits 1 s nach Reaktionsstart abgelesen; die Präzision war unter diesen Bedingungen nicht befriedigend).

Weitere Einstellungen: Syringe Plate 1:51 (5 $\mu 1$ Probe $+250 \mu 1$ Reagenz), Mode Selector Rate, Reaction Direction UP, Carouse Revolution 2, Decimal 0000., Factor 5000 (Ausdruck der Absorptionsdifferenz bei 340 und $380 \mathrm{~nm}$ ), Zero 0000 .

GEMSAEC

Rotoloader: Sample-Schalter B, Blank-Schalter WATER, Probe $10 \mu \mathrm{l}$, Verdünnungsmittel (bidest. Wasser) $50 \mu \mathrm{l}$, Reagenz $500 \mu 1$.

Analyzer und Control Modul: RATE, AUTO, IR $=10 \mathrm{~s}$ (entspricht $\mathrm{t}_{1}=35 \mathrm{~s}$ effektiv), $\mathrm{RI}=60 \mathrm{~s}, \mathrm{NR}=5$.

Computer: $\mathrm{IR}=10, \mathrm{RI}=60, \mathrm{NR}=5, \mathrm{SC}=0, \mathrm{KT}=0, \mathrm{TF}=35$, $\mathrm{TC}=5, \mathrm{AD}=4, \mathrm{CD}=0, \mathrm{HI}=0, \mathrm{LO}=0, \mathrm{SA}=1, \mathrm{RM}=1$, $X X=0$. Abweichungen von diesen Angaben ergeben sich bei Tabelle 3 und sind dort gekennzeichnet.

\section{Cobas Bio}

Pipettor: Probe $5 \mu \mathrm{l}$, Verdünnungsmittel (bidest. Wasser) $55 \mu \mathrm{l}$, Reagenz $250 \mu l$.

Analyżer: Faktor 1000, incubation time 0 , time of first reading $1 \mathrm{~s}$ (entspricht $t_{1}=5 \mathrm{~s}$ effektiv), time interval $300 \mathrm{~s}$, number of readings 2 .

\section{GILFORD 3500}

Dosierer: Probe $20 \mu \mathrm{l}$, Reagenz $1000 \mu \mathrm{l}$.

Kinetische Messüng (Abb. 11a): Meßbeginn $t_{1}=15 \mathrm{~s}$, Meßzeit $3 \times 13$ s.

Endpunktverfahren (Abb. 11b): Messung gegen den ReagenzienLeerwert nach $15 \mathrm{~min}$

\section{Experimentelle Angaben zu Tabelle 3}

Bei diesen Messungen wurde das Richtigkeitskontrollserum Kontrollogen ${ }^{\circledR}-\mathrm{L}$ der Behringwerke, Charge 3112 , verwendet IgG-Sollwert 10,42 g/l, bestimmt nach der Methode der einfachen radialen Immundiffusion mit Partigen ${ }^{\circledR}$-Pilatten der Behringwerke. Durch andere Auflösungen wurden zusätzlich die Konzentrationen 5,21 g/1 und 34,73 g/l hergestellt. Die drei 
Lösungen wurden 1:41 verdünnt als Probe in die Analyse eingesetzt.

Die Wiederfindung wurde durch Vergleich mit einer Verdünnungsreihe 1:6, 1:12, 1:24, 1:48 und 1:96 des T Protein-Standardserums ermittelt. Die Auswertung erfolgte über den Rechner TI 59 der Firma TEXAS INSTRUMENTS mit Statistik-Modul über eine parabolische Regression. Im Unterschied zu anderen Angaben in der Arbeit sind in Tabelle 3 Antigenkonzentrationen in der Probe angegeben.

Die Präzision in der Serie wurde direkt aus den gefundenen Absorptionsdifferenzen berechnet.

\section{Ergebnisse und Diskussion}

\section{Zeitabhängigkeit der Trübungskurve}

Die Reaktion von Proteinen mit ihren korrespondierenden Antikörpern führt in wäßriger Lösung zu einer zeitund konzentrationsabhängigen Trübung. Absorptionsmessungen, die vor Erreichen des Reaktionsendpunktes gegen den Reagenzien-Leerwert durchgeführt werden, ergeben ähnliche Abhängigkeiten wie bei der klassischen „Heidelberger-Kurve“. Die Art, wie die nach verschiedenen Reaktionszeiten aufgenommenen Kurven gegeneinander verschoben sind (Abb. 1), beweist jedoch, daß man bei kinetischen Systemen nicht von Antigen-, Antikörperüberschuß und Äquivalenz im Sinne der quantitativen Immunpräzipitation sprechen darf. Wir ersetzen daher im folgenden den Begriff „HeidelbergerKurve“ bewußt durch den Ausdruck „Trübungskurve“. Weiterhin charakterisieren wir das Verhältnis von Antigen- zu Antikörperkonzentration an Hand von Meßergebnissen, die sich in Annäherung an das End wertverfahren ergeben haben: unabhängig von der sonstigen Zusammensetzung des Reaktionsmediums wurden bei der durchgehend gewählten Antiserumverdünnung von 1:14,6 im Test (Ausnahme: Kurve 8b) Maxima zwischen etwa 40 und $70 \mathrm{mg} / 1 \mathrm{IgG}$ gefunden (vgl. Abb. 1c). Demzufolge werden wir bei Antigenkonzentrationen bis etwa $10 \mathrm{mg} / 1$ von ,,hohem Antikörperüberschuß", bei Antigenkonzentrationen zwischen etwa 10 und $40 \mathrm{mg} / 1$ von „mäßigem Antikörperüberschuß“, bei Antigenkonzentrationen $z$ wischen etwa 40 und $70 \mathrm{mg} / \mathrm{l}$ von „Äquivalenzbereich" und bei Konzentrationen über $70 \mathrm{mg} / 1$ von ,Antigenüberschuß" sprechen.

An Hand von Abbildung 1 ist zu erkennen, daß sich die Maxima der Trübungșkurven mit abnehmender Reaktionsdãuer nach liñks verschieben. Zugleich beginnt der aufsteigende Ast zunehmend rechts auf der Konzentrationșachse. Beide Effekte sind für quantitative Antigenbestimmungen ungünstig. Sie erklären sich aus der Rẹaktionskinetik (Abb. 2).

Bei mäßigem Antikörperüberschuß trübt sich die Lösung am schnellsten (Kurve b). Sowohl bei hohem Antikörperüberschuß (Kurve a) als auch im Äquivalenzbereich (Kurven $c$ und d) findet man größere lag-Phasen und geringere Reaktionsgeschwindigkeiten. Uber den Äquivalenzbereich hinaus nimmt die Trübungsgeschwindigkeit mit

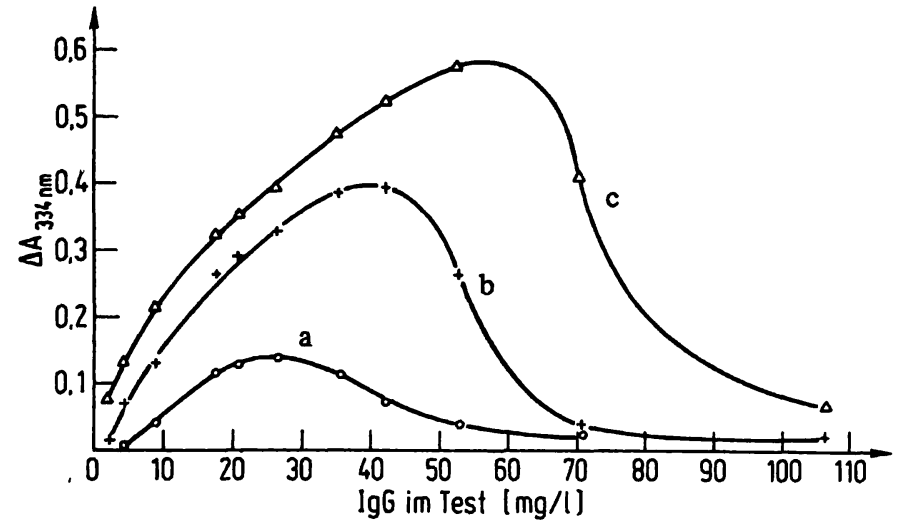

Abb. 1. Trübungskurven (a) $1 \mathrm{~min}$, (b) $5 \mathrm{~min}$, (c) $45 \mathrm{~min}$ nach Reaktionsstart gegen den Reagenzien-Leerwert gemessen. Reaktionsmedium: Antiserum 1:14,6 in isotonischer Natriumchlorid-Lösung. Messung bei $\mathrm{Hg} 334 \mathrm{~nm}$.

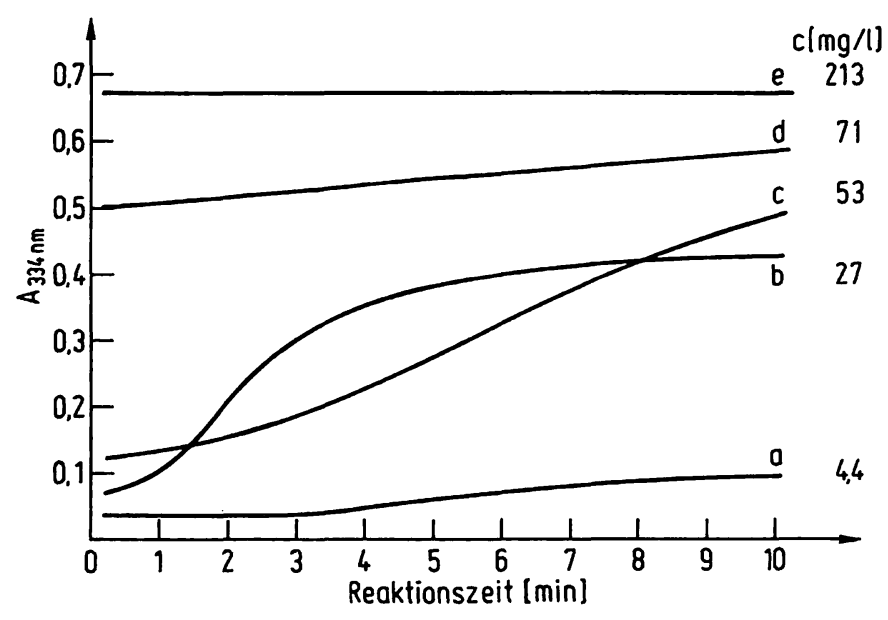

Abb. 2. Geschwindigkeit der Trübungszunahme mit steigender Antigenkonzentration. Reaktionsmedium: wie bei Abb. 1. Angegeben sind IgG-Testkonzentrationen. Die Anfangsabsorptionen wurden willkürlich gewählt. Messung bei $\mathrm{Hg} 334 \mathrm{~nm}$.

zunehmender Antigenkonzentration weiter ab bis schließlich kein Absorptionsanstieg mehr erfolgt (Kurve e).

Sieht man einmal von hohen Anfangsgeschwindigkeiten $a b$, die bei starkem Antigenüberschuß unter Verwendung der stopped-flow-Mischkammer beobachtet wurden $(1,19)$, stịmmen die in Abbildung 2 dargestellten Verhältnisse mit allen übrigen Literaturergebnissen überein, wie sie teils bei nephelometrischen Untersuchungen (2-4), teils turbidimetrisch an anderen Antigen-Antikörper-Systemen (15) gefunden wurden. Sie dürften somit für alle Antigen-Antikörper-Reaktionen in unterschiedlichen Reaktionsmedien typisch sein (vigl. auch die folgenden Abbildungen 4 und 5).

Anhand der Abbildung 2 erkennt man nun leicht, daß bei geringen Antigenkonzentrationen ein kleines Meßintervall nach dem Reaktionsstart in den Bereich der lag-Phasen fallt und die Trübungskurve auf der Konzentrationsachse daher rechtsverschoben bzw. s-förmig (9) beginnen muß (Abb. 1). Da die Trübung auch bei höhe- 
ren Antigenkonzentrationen wieder stärker verzögert einsetzt, erklärt sich auch die Linksverschiebung der Maxima mit abnehmender Reaktionsdauer.

Die Abbildung 1 und auch der folgenden Abbildung 6 zugrundeliegende Messung gegen den Reagenzien-Leerwert stellt $\mathrm{zwar}$ keine typische kinetische Technik dar, kann jedoch als Grenzfall der fixed-time-Messung $\left(t_{1}=0 \mathrm{~s}\right)$ betrachtet werden. Sie ist typisch für Bedingungen, bei denen die Messung unabhängig von der Antigenkonzentration in einer sehr frühen Reaktionsphase beginnt. Es sei an dieser Stelle darauf hingewiesen, daß es sich teilweise einzubürgern beginnt, fixedtime-Messungen, bei denen die Reaktion am Meßbeginn noch nicht wesentlich abgelaufen ist und bei denen am Ende des Meßintervalls der Endpunkt weitgehend erreicht ist, den Endwertverfahren zuzurechnen. Wir werden im folgenden jedoch Messungen, die nach dem Reaktionsstart beginnen und bei denen der Endpunkt wie in Abbildung la oder $1 \mathrm{~b}$ noch deutlich unterschritten ist, als kinetische Techniken bezeichnen.

Abbildung 3 stellt nun Trübungskurven dar, bei denen der Meßzeitraum (mitbedingt durch den Zusatz von Polyethylenglykol, siehe weiter unten) in weiter fortgeschrittene Reaktionsphasen fällt.

Kurve 3a zeigt zunächst noch einmal den für Messungen im Bereich der Anlaufphasen typischen, bereits diskutierten rechtsverschobenen Anlauf der Trübungskurve. Mit steigender Reaktionszeit $t_{1}$ verlagert sich der Beginn der Trübungskurve erwartungsgemäß nach links; zugleich werden die Absorptionsdifferenzen allgemein größer. Mit weiter zunehmender Reaktionszeit folgt eine bereits von Ebeling (8) beschriebene weitgehende Verflachung der Trübungskurve, und das Maximum verschiebt sich nach rechts (Abb. 3c). Auffällig an Kurve $3 c$ ist weiterhin ein Wendepunkt im Bereich des mäßigen Antikörperüberschusses. Die Ursache dieses in der Tur-

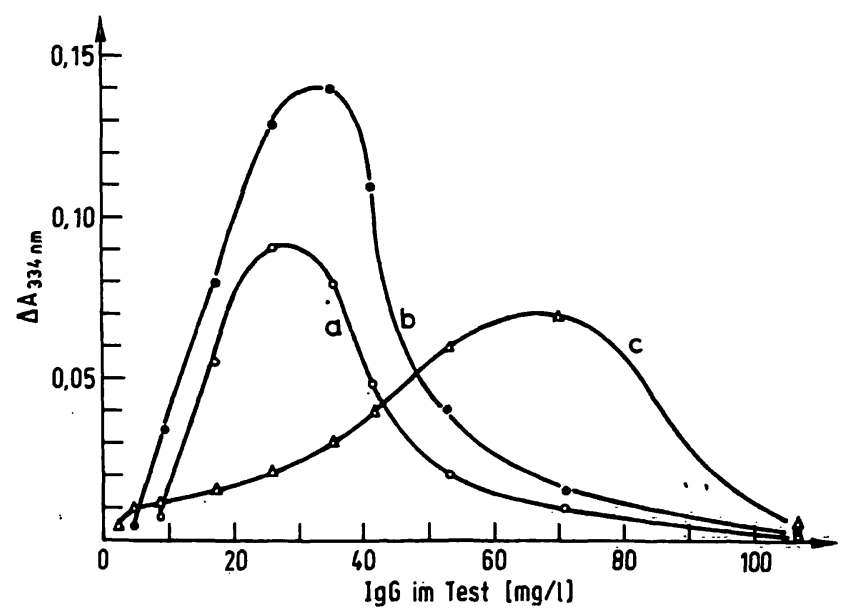

Abb. 3. Trübungskurven bei einem konstanten fixed-time-Intervall $t_{2}-t_{1}=12 \mathrm{~s}$ nach (a) $t_{1}=6 \mathrm{~s}$, (b) $t_{1}=12 \mathrm{~s}$, (c) $t_{1}=60$ s. Reaktionsmedium: $9 \mathrm{~g} / \mathrm{l} \mathrm{NaCl}, 10 \mathrm{~g} / \mathrm{l}$ Polyethylenglykol, Antiserum 1:14,6. Messung bei $\mathrm{Hg} 334 \mathrm{~nm}$. bidimetrie unerwünschten Effektes wird weiter unten diskutiert.

Ein Vergleich der in Abbildung 3 dargestellten Trübungskurven zeigt, daß immunturbidimetrische Messungen in einem gewissen Rahmen durch zeitliche Verschiebung des Meßzeitraumes optimiert werden können. In der Praxis wird man allerdings einen frühen Meßbeginn anstreben und das Reagenz entsprechend modifizieren (vgl. den weiter unten folgenden Text).

\section{Be schleunigung der Immunpräzipitation}

Da die Geschwindigkeit von Immunreaktionen von der Ionenstärke, Polymerzusätzen und anderen Faktoren abhängt, muß der Einfluß von Meßbeginn und Meßzeitraum im Zusammenhang mit dem Reaktionsmedium gesehen werden. Es finden sich in der Literatur teilweise wiedersprüchliche Auffassungen, wieweit das Reaktionsmedium über die Reaktionsgeschwindigkeit hinaus den Endwert beeinflußt $(4,10,13,17,18,21,26,29)$. Wir werden auf diese Frage an späterer Stelle zurückkommen.

Wie ein Vergleich der Abbildungen 2 und 4 zeigt, erhöht bereits ein Zusatz von $10 \mathrm{~g} / 1$ Polyethylenglykol 6000 die Geschwindigkeit der Trübungszunahme merklich.

Besonders auffällig an diesem bekannten Effekt $(1,3,4$, 20-27) sind starke Absorotionszunahmen im Äquivalenzbereich und bei nicht zu hohem Antigenüberschuß.

Auch bei Herabsetzung der Elektrolytkonzentration verlaufen Immunreaktionen im allgemeinen schneller. In destilliertem Wasser werden bemerkenswert hohe Geschwindigkeiten erreicht (Abb. 5).

$\mathrm{Da}$ in Gegenwart von Serumproben bisweilen unspezifische Fällungen auftreten (15), ist entionisiertes Wasser jedoch kein geeignetes Reaktionsmedium. Bei zu geringer Ionenstärke treten unspezifische Fällungen in Gegen-

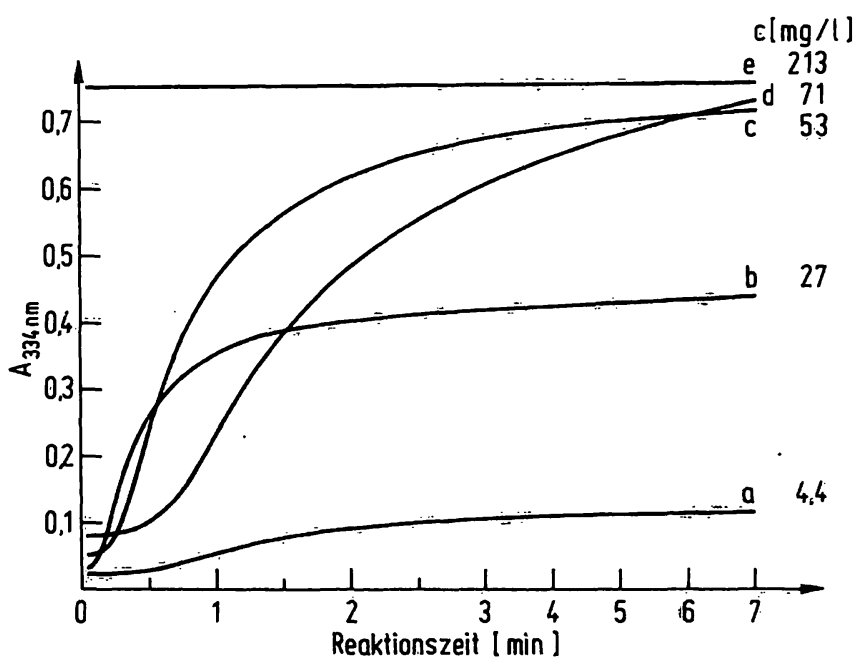

Abb. 4. Geschwindigkeit der Trübungszunahme bei steigender Antigenkonzentration in isotonischer NatriumchloridLösung mit Zusatz von $10 \mathrm{~g} / 1$ Polyethylenglykol 6000 . Alle anderen Bedingungen entsprechen den Angaben zu Abb. 2. 


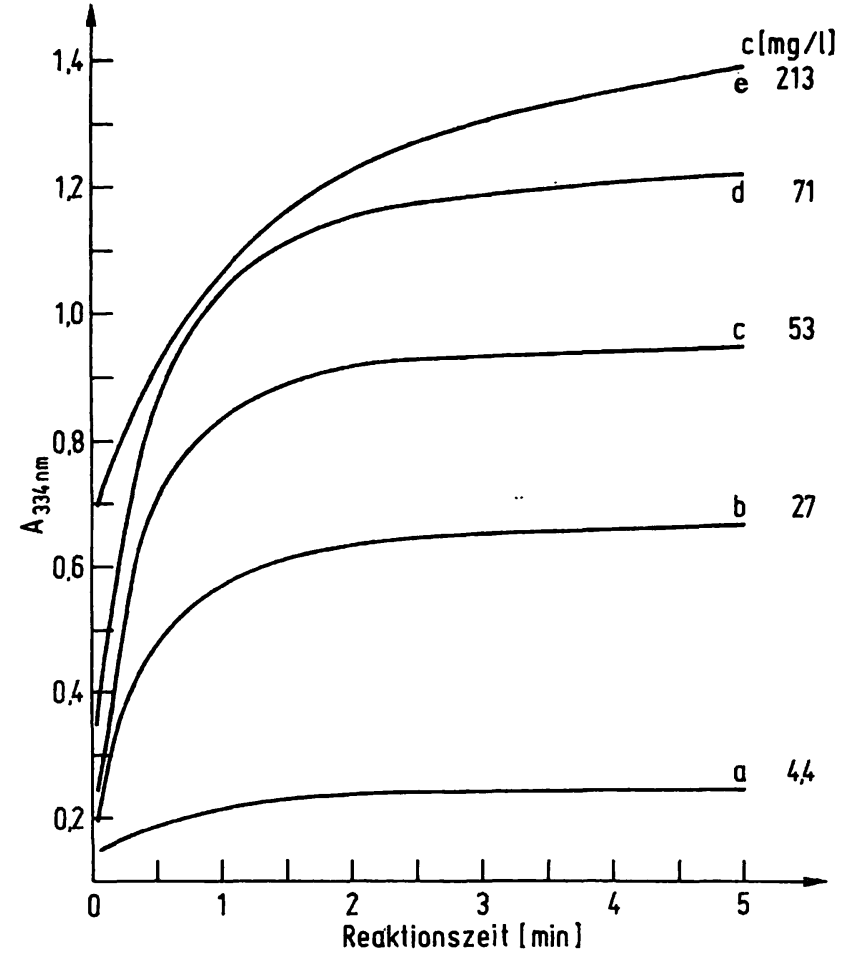

Abb. 5. Geschwindigkeit der Trübungszunahme bei verschiedenen Antigenkonzentrationen in bidest. Wasser. Alle anderen Bedingungen entsprechen den Angaben zu Abb. 2.

wart von Polyethylenglykol oder verwandter Polymerer noch vermehrt auf. Deshalb muß gerade bei Verwendung von Polyethylenglykol oder ähnlicher Zusätze auf eine hinreichend hohe Ionenstärke geachtet werden; auch durch nachträgliche Zugabe geeigneter Salze lassen sich unspezifische Fällungen meist wieder weitgehend auflösen. Die durch den Polymerzusatz angestrebte reaktionsbeschleunigende Wirkung geht durch gleichzeitigen Salzzusatz allerdings wieder mehr oder minder verloren.

Sofern unspezifische Ausfallungen vermieden werden, ist bei Messungen gegen den Reagenzien-Leerwert bzw. bei Endpunktverfahren im Hinblick auf eine Verkürzung der Meßdauer die Zugabe von Beschleunigungsmitteln sinnvoll.

Auch bei kinetischen fixxèd-time-Messungen werden häufig Polymerbeschleuniger verwendet $(1,5,7,9-13,26)$. Wie ein Vergleich der Abbildungen 1 und 6 zeigt, lassen sich durch solche Zusätze unter geeigneten Bedingungen in kuržen Meßzzeiten günstige Trübungskurven erreichen. Bei kinetischen fixed-time-Messungen ist jedoch zu bẹachten, dạß die Eintrübung der Meß̣lösung bei bestimmten Antigenkonzentrationen außerordentlich schnell einsetzen kann (vgl. 1. c. (3)). Bereits wenige Sekunden zwischen Reaktionsstart und Meßbeginn können genügen, um primäre Trübungszunahmen nicht mehr zu erfassen. Da die Immuntrübung grund sätzlich bei mäßigem Antikörperüberschuß am schnellsten abläuft (vgl. hierzu die Abb. 2, 4 und 5), ist der „Verlust" hier am größten. Er wirkt sich meist durch einen ver-

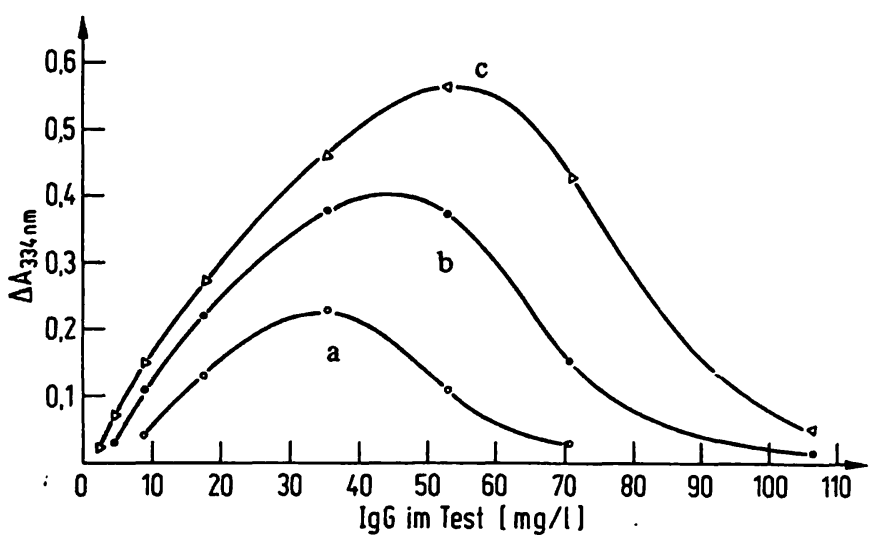

Abb. 6. Trübungskurven (a) $30 \mathrm{~s}$, (b) $60 \mathrm{~s}$, (c) $120 \mathrm{~s}$ nach Reaktionsstart. Bis auf den Zusatz von $20 \mathrm{~g} / 1$ Polyethylenglykol 6000 sind die sonstigen Bedingungen denen der Abb. 1 analog.

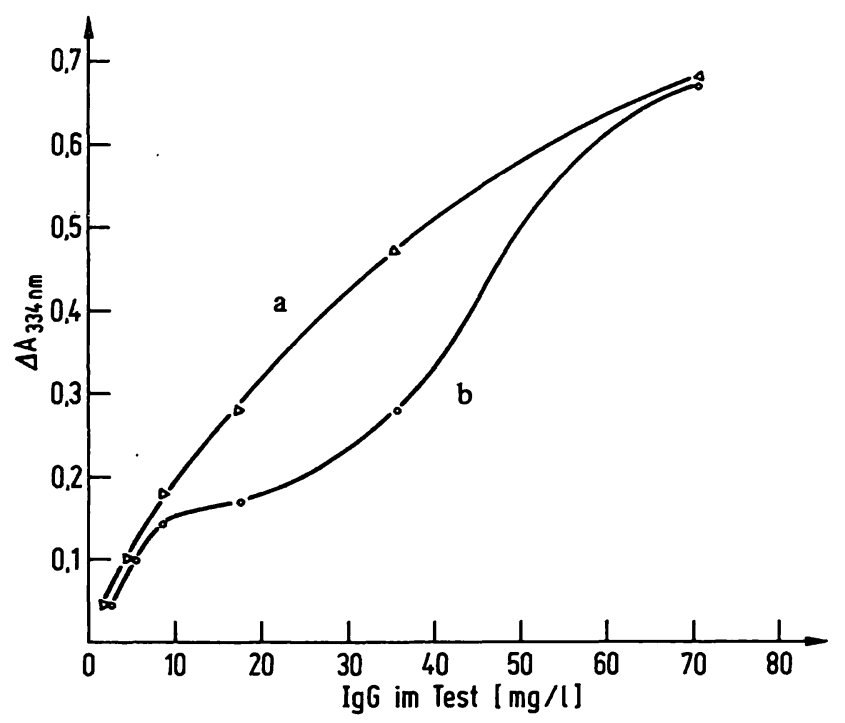

Abb. 7. Verformung deș aufsteigenden Astes der Trübungskurve, bedingt durch schnelle Eintrübung und späten Meßbeginn. Kurve a: $t_{1}=15 \mathrm{~s}, t_{2}=5 \mathrm{~min} ;$ Kurve $b$ : $t_{1}=30 \mathrm{~s}, t_{2}=5 \mathrm{~min}$.

Reaktionsmedium: $9 \mathrm{~g} / 1 \mathrm{NaCl}, 10 \mathrm{~g} / 1$ Polyethylenglykol, Antiserum 1:14,6. Messung bei $\mathrm{Hg} 334 \mathrm{~nm}$.

minderten Anstieg im Bereich des aufsteigenden Astes der Trübungskurve aus. Je nach Meßbeginn, Meßzeitraum und Reaktionsgeschwindigkeit entstehen ausgeprägte s-förmige Bezirke mit Wendepunkt wie in Abbildung 7 oder auch nur geradlinige Verläufe. Es sei in diesem Zusammenhang erwähnt, daß bei extrem erhöhter Antiserumkonzentration, wie sie normalerweise nicht zur Antigenbestimmung verwendet wird, zwei Maxima der Trụ̈bungskurve auftreten können, von denen das rechte unter Umständen unbeobachtet bleibt, wenn hinreichend hohe Antigenkonzentrationen nicht mehr zur Verfügung stehen. In dem kritischen Bereich des mäßigen Antikörperüberschusses ist die Reaktionsgeschwindigkeit dann so hoch, daß die Trübung vor Beginn der Messung weitgehend ihren Endpunkt erreicht hat (inner- 
halb der ersten 10 Sekunden wurden Absorptionszunahmen größer 1 beobachtet). Bei einer Herabsetzung der Reaktionsgeschwindigkeit bzw. der Antiserumkonzentration verschwindet das linke der beiden Maxima wieder.

Da die Trübungskurve in allen Fällen durch einfache Vorverlegung des Meßbeginns wieder ihre stetig gekrümmte Form erhält, ist die von Malkus et al. (9) angenommene abgestufte Immunaggregation als Ursache der vorstehend beschriebenen Effekte auszuschließen. Es sei am Rande erwähnt, daß sich aus dem Zusammenspiel von Meßzeitraum und Reaktionsgeschwindigkeit möglicherweise unterschiedliche Ergebnisse bei der Ermittlung von Reaktionsordnungen immunologischer Trübungsreaktionen erklären (4). Der Wert der Angabe von Reaktionsordnungen ist bei der optischen Verfolgung von Immunpräzipitationen angesichts der komplexen Vorgänge insgesamt fraglich. So ändert sich mit der Größe der Immunkomplexe auch die Streulichtgeometrie. Diese Änderung wird von unterschiedlichen Meßgeräten unterschiedlich erfaßt.

Wenn es um die Ermittlung von Antigenkonzentrationen innerhalb kleiner Meßbereiche geht oder graphisch ausgewertet wird, kann eine Linearisierung der Referenzkurve durchaus nützlich sein. Liegen bei einer Erweiterung des Meßbereiches lineare und gekrümmte Bezirke nebeneinander vor, wird die rechnerische Auswertung über einfache parabolische Regressionen ungenau. Es muß dann entweder eine geeignete Interpolationsfunktion (,spline function“) herangezogen oder graphisch ausgewertet werden. Bei stärkeren Verformungen hierzu können auch chargenbedingte Aviditäts- und/ oder Titerunterschiede der Antiseren beitragen - ist die Auswertung quantitativer Messungen naturgemäß immer gestört. Abgesehen davon, daß ein verminderter $\mathrm{An}$ stieg $\Delta \mathrm{A} / \Delta \mathrm{c}$ eine Differenzierung zwischen ver schiedenen Antigenkonzentrationen erschwert, werden solche Kurven innerhalb eines größeren Meßbereiches nur noch von aufwendigen Rechenprogrammen beschrieben.

\section{Verzögerung der Immunpräzipitation}

Bei der praktischen Durchführung immunturbidimetrischer fixed-time-Messungen ist der frühest mögliche Meßbeginn von den gerätetechnischen Voraussetzungen abhängig. Die Immuntrübung muß so langsam einsetzen, daß eine störende Verformung der Referenzkurve vermieden wird. Die Verringerung der Reaktionsgeschwindigkeit gelingt entweder durch Fortlassen von Beschleunigungsmitteln und/oder Einsatz von Hemmitteln. Neben Elektrolyten kommen auch verschiedene niedermolekulare organische Verbindungen $(16,17)$ als Hemmmittel in Betracht.

Eine mäßige Verlangsamung der Immunpräzipitation kann, wenn der frühe Teil der Eintrübung dadurch vollständiger erfaßt wird, in Teilbereichen der Referenzkurve zu erheblichen Vergrößerungen der gemessenen
Absorptionsdifferenzen führen. In anderen Konzentrationsbereichen ist dagegen - konstante Zeiten $t_{1}$ und $t_{2}$ vorausgesetz $t$ - mit der Herabsetzung der Reaktionsgeschwindigkeit auch eine Reduzierung der Empfindlichkeit sowie eine Linksverschiebung des Maximums der Trübungskurve verbunden.

Hinsichtlich der Wirkung von Elektrolyten stimmen wir mit früheren Ergebnissen von Hawkins (15) darin überein, daß vielfach die Geschwindigkeit der Reaktion stärker als der Endpunkt beeinflußt wird. Insbesondere finden wir bei hohem bis mäßigem Antikörperüberschuß in verschiedenen Reaktionșmedien kaum Unterschiede im Endpunkt (Tab. 1).

Bei Antigenüberschuß treten jedoch neben größeren Unterschieden in der Reaktionsgeschwindigkeit offensichtlich auch unterschiedliche Endpunkte auf (Tab. 2).

Derartige Aussagen sind insofern problematisch, als es bei fortgesetztem Stehen der Ansätze zu șekundären Umwandlungen der Präzipitate kommen kann (Ausflockungen etc.). Es ist dann nicht immer sicher festzustellen, wieweit sich die Absorptionen der verschiedenen Ansätze letżtlich angleichen würden. Muß die Eintrübung aus gerätetechnischen Gründen verzögert werden, erlaubt die Wirkung der Elektrolyte, soweit sie nur kinetisch geprägt ist, durch entsprechende Verlängerung der Meßzeit die Empfindlichkeit schnellerer Reaktionen wiederherzustellen.

Darüber hinaus ist es in Verbindung mit einer Erhöhung der Antiserumkonzentration sogar möglich, die Empfindlichkeit trotz einer Verringerung der Reaktionsge-

Tab. 1. Endpunkt der Trübung in verschiedenen Reaktionsmedien bei einer IgG-Konzentration $17,8 \mathrm{mg} / \mathrm{l}$ im Test. Antiserum 1:14,6. Messung gegen den Reagenzien-Leerwert bei $340 \mathrm{~nm}$. Unspezifische Absorptionen sind korrigiert.

\begin{tabular}{|c|c|c|c|c|}
\hline \multirow[t]{2}{*}{ Reaktionsmedium } & \multicolumn{2}{|c|}{ Reaktionsdauer } & \multirow[b]{2}{*}{$60 \mathrm{~min}$} & \multirow[b]{2}{*}{$120 \mathrm{mir}$} \\
\hline & $15 \mathrm{~min}$ & $30 \mathrm{~min}$ & & \\
\hline Wasser & 0,350 & 0,346 & 0,348 & 0,353 \\
\hline $9 \mathrm{~g} / 1$ Natriumchlorid & 0,320 & 0,325 & 0,330 & 0,332 \\
\hline $\begin{array}{c}9 \mathrm{~g} / 1 \text { Natriumchlorid } \\
10 \mathrm{~g} / \mathrm{l} \text { Polyethylen- } \\
\text { glykol } 6000\end{array}$ & 0,325 & 0,331 & 0,334 & 0,345 \\
\hline $\begin{array}{c}9 \mathrm{~g} / 1 \text { Natriumchlorid } \\
20 \mathrm{~g} / 1 \text { Polyethylen- } \\
\text { glykol } 6000\end{array}$ & 0,329 & 0,330 & 0,337 & 0,340 \\
\hline $\begin{array}{c}9 \mathrm{~g} / 1 \text { Natriumchlorid } \\
50 \mathrm{~g} / 1 \text { Polyethylen: } \\
\text { glykol } 6000\end{array}$ & 0,387 & 0,393 & 0,399 & 0,411 \\
\hline $\begin{array}{l}9 \mathrm{~g} / \mathrm{l} \text { Natriumchlorid } \\
10 \mathrm{~g} / 1 \text { Dimethylsulfoxid }\end{array}$ & 0,300 & 0,311 & 0,314 & 0,329 \\
\hline $\begin{array}{l}9 \mathrm{~g} / 1 \text { Natriumchlorid } \\
10 \mathrm{~g} / 1 \text { Dioxan }\end{array}$ & 0,300 & 0,316 & 0,323 & 0,337 \\
\hline $\begin{array}{l}\text { phosphatgepufferte } \\
\text { Natriumchlorid-Lösung }\end{array}$ & 0,291 & 0,296 & 0,305 & 0,314 \\
\hline $\begin{array}{l}\text { phosphatgepufferte } \\
\text { Natriumchlorid-Lösung } \\
20 \text { g/1 Polyethylen- } \\
\text { glykol } 6000\end{array}$ & 0,327 & 0,331 & 0,335 & 0,343 \\
\hline
\end{tabular}


Tab. 2. Absorptionszunahme in verschiedenen Reaktionsmedien bei sehr hoher IgG-Konzentration von $213 \mathrm{mg} / 1 \mathrm{im}$ Test. Antiserum 1:14,6, Messung gegen den Reagenzien-Leerwert bei $\mathrm{Hg} 334 \mathrm{~nm}$. Unspezifische Absorptionen sind korrigiert.

\begin{tabular}{|c|c|c|c|c|}
\hline \multirow[t]{2}{*}{ Reaktionsmedium } & \multicolumn{4}{|c|}{ Reaktionsdauer } \\
\hline & $30 \mathrm{~min}$ & $60 \mathrm{~min}$ & $120 \mathrm{~min}$ & $\begin{array}{l}1080 \\
\min \end{array}$ \\
\hline Wasser & 0,767 & 0,804 & 0,826 & 0,870 \\
\hline $9 \mathrm{~g} / 1$ Natriumchlorid & 0,006 & 0,004 & 0,008 & 0,150 \\
\hline $\begin{array}{c}9 \mathrm{~g} / 1 \text { Natriumchlorid } \\
10 \mathrm{~g} / 1 \text { Polyethylen- } \\
\text { glykol } 6000\end{array}$ & 0,150 & 0,287 & 0,391 & 0,550 \\
\hline $\begin{array}{c}9 \mathrm{~g} / 1 \text { Natriumchlorid } \\
20 \mathrm{~g} / 1 \text { Polyethylen- } \\
\text { glykol } 6000\end{array}$ & 0,687 & 0,748 & 0,786 & 0,860 \\
\hline $\begin{array}{c}9 \mathrm{~g} / 1 \text { Natriumchlorid } \\
50 \mathrm{~g} / 1 \text { Polyethylen- } \\
\text { glykol } 6000\end{array}$ & 1,321 & 1,285 & 1,250 & 1,200 \\
\hline $\begin{array}{l}9 \mathrm{~g} / 1 \text { Natriumchlorid } \\
10 \mathrm{~g} / 1 \text { Dimethylsulfoxid }\end{array}$ & 0,002 & 0,002 & 0,002 & 0,110 \\
\hline $\begin{array}{l}9 \mathrm{~g} / 1 \text { Natriumchlorid } \\
10 \mathrm{~g} / 1 \text { Dioxan }\end{array}$ & 0,002 & 0,002 & 0,003 & 0,120 \\
\hline $\begin{array}{l}\text { phosphatgepufferte } \\
\text { Natriumchlorid-Lösung }\end{array}$ & 0,002 & 0,002 & 0,003 & 0,030 \\
\hline $\begin{array}{l}\text { phosphatgepufferte } \\
\text { Natriumchlorid-Lösung } \\
20 \text { g/l Polyethylen- } \\
\text { glykol } 6000\end{array}$ & 0,286 & 0,424 & 0,514 & 0,610 \\
\hline
\end{tabular}

schwindigkeit zu steigern (vgl. die Kurven Abb. 8a und b). Dieser Effekt tritt allerdings nicht bei hohem Antikörperüberschuß ein, da hier eine zusätzliche Anhebung der Antiserumkonzentration weder die Trübungsgeschwindigkeit noch den Endpunkt wesentlich beeinflußt.

Dies erklärt im übrigen auch, warum Chargenschwankungen der Antiseren sich bei hohem Antikörperüberschuß kaum bemerkbar machen. Erst mit steigendem Antigenanteil führt eine Anhebung der Antiserumkonzentration zum höheren Endpunkt. Wird unter diesen Verhältnissen die Reaktionsgeschwindigkeit durch Salzzusatž redužiert, entstehen wie in Abbildung $8 \mathrm{~b}$ mit geringer Geschwindigkeit hohe Trübungen. Der gleiche Effekt wäre nicht erreichbar, wollte man versuchen, die Wirkung kleiner Antiserumkonzentrationen durch Polymerzusatz zu verstärken.

\section{Praktische Beispièle}

In der Praxis wird man einen möglichst frühen Meßbeginn $t_{1}$ anstreben, um durch Beschleunigung der Trübungsreaktion kurze Meßzeiten zu erzielen. Bei sequentieller Messung ist dies weit wichtiger aḷ bei paralleler.

Unter den Routinemeßgeräten findet man allerdings nur wenige, die einen wirklich frühen Meßbeginn erlauben. $\mathrm{Zu}$ ihnen gehören die Zentrifugalanalysatoren CentrifiChem und Cobas Bio, die eine präzise Messung bereits 5 Sekunden nach Reaktionsstart gestatten. Wegen des parallelen Meßprinzips ermöglichen beide Geräte zudem die Wahl eines größeren Meßintervalls und bie-

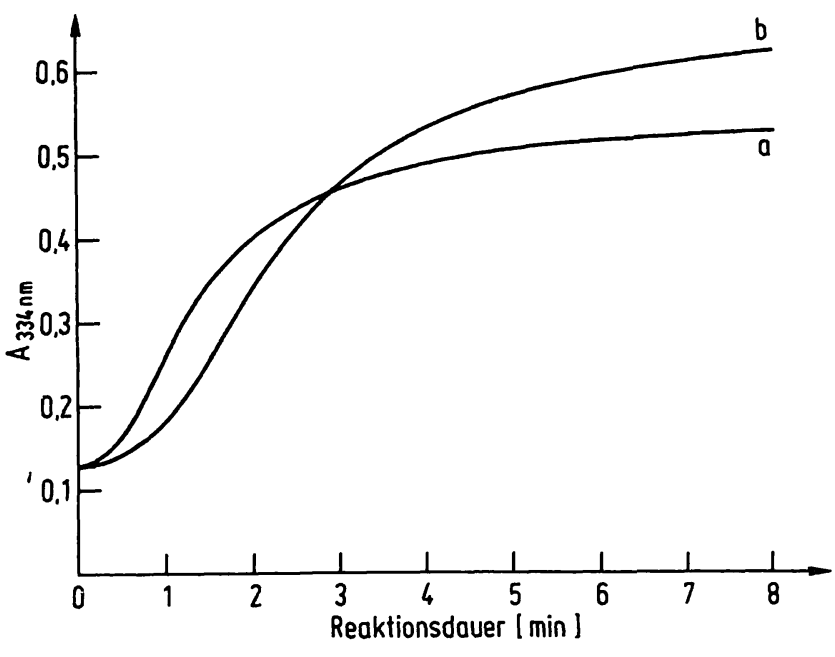

Abb. 8. Vergleich der Reaktionskinetik bei verschiedenen Salzund Antiserumkonzentrationen: (a) Antiserum 1:14,6 in $9 \mathrm{~g} / 1 \mathrm{NaCl}$, (b) Antiserum $1: 6$ in $30 \mathrm{~g} / 1 \mathrm{NaCl}$. Antigenkonzentration jeweils $26,6 \mathrm{mg} / 1 \mathrm{im}$ Test. Messung bei $\mathrm{Hg} 334 \mathrm{~nm}$.

ten damit in doppelter Hinsicht günstige Voraussetzungen für immunturbidimetrische fixed-time-Messungen.

Infolge der hohen Zentrifugalbeschleunigung werden die entstandenen Immunkomplexe bei beiden Geräten abzentrifugiert. Beim longitudinal messenden Cobas Bio ändert sich hierdurch die Absorption kaum. Beim CentrifiChem beginnt die Absorption dagegen nach einer Reaktionszeit von etwa 5 Minuten wieder abzusinken $(8,30$, vgl. auch 1. c. (25)), wenn die Geschwindigkeit der Trübungsabnahme durch die Zentrifugation größer wird als die Geschwindigkeit der Trübungszunahme durch die Immunreaktion. Dieser Effekt ist bei mäßigem Antikörperüberschuß am ausgeprägtesten. Zentrifugiert man ausreagierte Lösungen, findet man in diesem Meß̧bereich zunächst einen starken, bald jedoch nur noch einen langsamen Absorptionsabfall (Abb. 9).

Dieser biphasische Verlauf und die Tatsache, daß die Absorptionen nicht bis auf den Reagenzien-Leerwert absinken, sprechen für die Vorstellung, daß bei mäßigem Antikörperüberschuß ein heterogenes Gemisch mit einem Anteil besonders großer Immunkomplexe vorliegt (vgl. l. c. (4)).

Bei einem weiteren Zentrifugalanalysator, dem GEMSAEC der Firma Electro Nucleonics, liegt der frühestmögliche Meßbeginn $t_{1}$ effektiv bei etwa $35 \mathrm{Se}$ kunden. Bei zu hoher Beschleunigung der Immunreaktion kommt es daher zu den in Abbildung 10 dargestellten Verformungen der Referenzkurve. Zudem ergeben sich als Folge hoher Reaktionsgeschwindigkeiten in diesem Bereich erfahrungsgemäß Meßschwankungen. Im Vergleich zu den Bedingungen am Centrifichem und Cobas Bio muß die Eintrübung am GEMSAEC daher verlangsamt werden.

Um dennoch günstige Maxima der Trübungskurve und ausreichende Empfindlichkeiten bei geringen Antigen- 


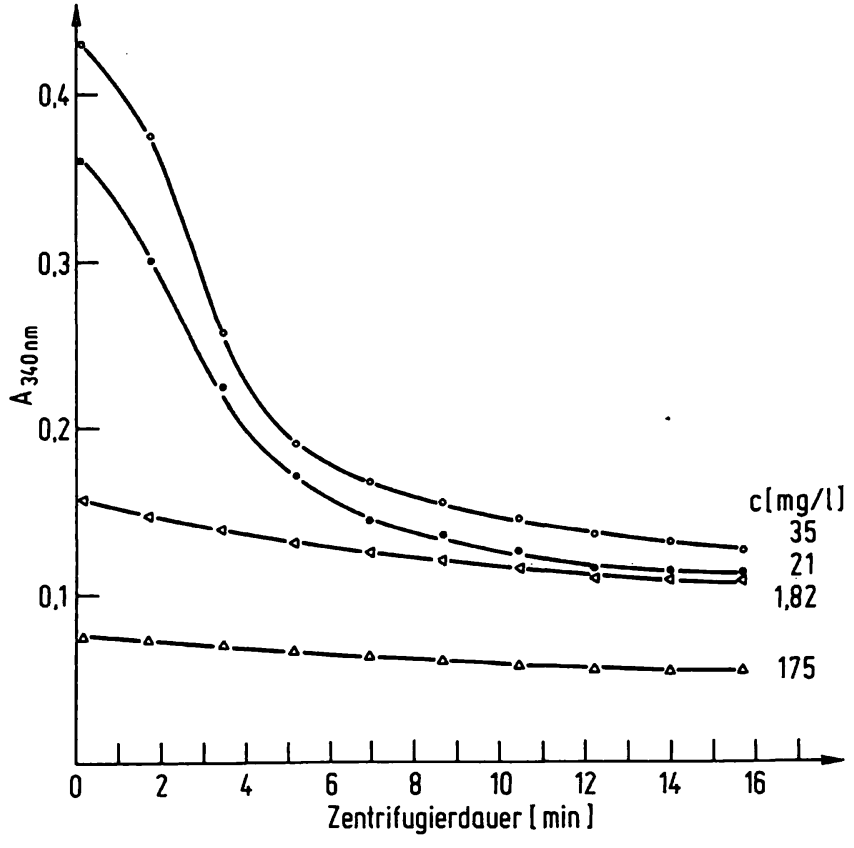

Abb. 9. Verminderung der Absorption am CentrifiChem durch Zentrifugation zuvor ausreagierter Lösungen bei vier Antigenkonzentrationen. Messung bei $340 \mathrm{~nm}$.

konzentrationen zu erreichen, sollte $t_{2}$ so groß wie möglich gewählt werden. In Tabelle 3 sind Vergleichsmessungen am GEMSAEC aufgeführt, bei denen $t_{2}$ versuchsweise vergrößert und die Reaktionsgeschwindigkeit durch Erhöhung der Natriumchlorid-Konzentration herabgesetzt wurde. Es zeigt sich, daß die Präzision der Messung besser wird. Es ist anzumerken, daß wegen der geringeren Drehgeschwindigkeit und dem vergleichsweise geringen Abstand der Küvetten vom Drehmittelpunkt am GEMSAEC kaum ein Abzentrifugieren von Immunkomplexen beobachtet wird, so daß längere Reaktionszeiten als am CentrifiChem realisierbar sind. Alternativ zur kinetischen fixed-time-Technik gestattet der GEMSAEC Messungen gegen den Reagenzien-Leer- wert $(23,24,31)$, der bei diesem Gerät empfehlenswertesten Technik, wenn große Meßbereiche verwendet werden sollen. Bei solchen Messungen existiert die Gefahr der Verformung der Referenzkurve durch zu hohe Anfangsgeschwindigkeiten naturgemäß nicht. Im Falle der Serum-IgG-Bestimmung kann auf einen gesonderten Lauf zur Korrektur des Proben-Leerwertes verzichtet werden, so daß der Arbeitsaufwand nicht höher als bei kinetischen Messungen ist.

Hinsichtlich der Parallelmessung mehrerer Proben ähnelt der ABA-100 den vorstehenden Zentrifugalanalysatoren. Für immunturbidimetrische fixed-time-Messungen eignet sich das Programm FRR (First Revolution Read). In der sogenannten Normalposition des Photometers kann ein erster Meßpunkt bereits innerhalb der ersten Sekunde nach dem Reaktionsstart aufgenommen werden. Die praktische Erfahrung hat jedòch gezeigt, daß die Präzision der Messungen durch Verzögerung des Meßbeginns verbessert werden kann. Der nächstmögliche Meßbeginn liegt bei $t_{1}=10$ Sekunden. Infolge dieser ansich geringfügigen Verzögerung des Meßbeginns darf die Reaktion nicht mehr so stark wie am CentrifiChem oder Cobas Bio beschleunigt werden. Es ist dem relativ großen Meßintervall von 10 Minuten zu verdanken, daß trotz der Herabsetzung der Reaktionsgeschwindigkeit und trotż des der Turbidimetrie entgegenstehenden bichromatischen Meßprinzips auch bei Verwendung des Routinefilters 340/380 nm noch ausreichende Empfindlichkeit erzielt werden (Abb. 10).

Durch Verwendung des speziellen Filters 340/650 nm läßt sich die Empfindlichkeit etwa um den Faktor 4 steigern.

$\mathrm{Zu}$ den Vertretern sequentieller Meßtechnik gehört der Gilford 3500. Um bei dieser Geräteklasse einen nach heutigen Maßstäben ausreichenden Probendurchsatz zu erreichen, muß spätestens nach 60 Sekunden ein Probenwechsel erfolgen. Da beim Gilford 3500 für Dosierung, Transfer der Meßlösung und Temperaturanpassung

Tab. 3. Präzision in der Serie und Wiederfindung bei verschiedenen Analysenautomaten und Meßtechniken.

\begin{tabular}{|c|c|c|c|c|c|c|c|c|c|}
\hline \multirow[t]{3}{*}{ Gerät } & \multicolumn{9}{|c|}{ Präzision und Wiederfindung } \\
\hline & \multicolumn{3}{|c|}{ Soll: $5,21 \mathrm{~g} / 1$} & \multicolumn{3}{|c|}{ Soll: $10,42 \mathrm{~g} / 1$} & \multicolumn{3}{|c|}{ Soll: $34,73 \mathrm{~g} / 1$} \\
\hline & $\begin{array}{l}\text { VK } \\
(\%)\end{array}$ & $\mathrm{n}$ & $\begin{array}{l}\text { Wieder- } \\
\text { findung } \\
(\mathrm{g} / \mathrm{l})\end{array}$ & $\begin{array}{l}\text { VK } \\
(\%)\end{array}$ & $\mathbf{n}$ & $\begin{array}{l}\text { Wieder- } \\
\text { findung } \\
\text { (g/l) }\end{array}$ & $\begin{array}{l}\text { VK } \\
(\%)\end{array}$ & $\mathbf{n}$ & $\begin{array}{l}\text { Wieder- } \\
\text { findung } \\
\text { (g/l) }\end{array}$ \\
\hline $\begin{array}{l}\text { ABA-100 } \\
\text { CentrifiChem } \\
\text { Cobas Bio } \\
\text { GEMSAEC } \\
\text { GEMSAEC* } \\
\text { Gilford 3500** } \\
\text { Gilford } 3500 * * *\end{array}$ & $\begin{array}{r}5,2 \\
6,0 \\
2,9 \\
14,5 \\
3,3 \\
5,3 \\
4,3\end{array}$ & $\begin{array}{l}20 \\
20 \\
20 \\
15 \\
15 \\
20 \\
15\end{array}$ & $\begin{array}{l}5,58 \\
6,43 \\
5,43 \\
5,57 \\
6,20 \\
5,39 \\
6,17\end{array}$ & $\begin{array}{r}2,3 \\
2,8 \\
1,9 \\
10,9 \\
6,0 \\
1,2 \\
1,2\end{array}$ & $\begin{array}{l}20 \\
20 \\
20 \\
15 \\
15 \\
20 \\
15\end{array}$ & $\begin{array}{l}11,18 \\
10,60 \\
10,69 \\
12,39 \\
11,90 \\
10,41 \\
11,07\end{array}$ & $\begin{array}{r}6,5 \\
5,9 \\
3,6 \\
20,2 \\
9,7 \\
2,1 \\
2,6\end{array}$ & $\begin{array}{l}20 \\
20 \\
20 \\
15 \\
15 \\
20 \\
15\end{array}$ & $\begin{array}{l}34,45 \\
34,13 \\
32,79 \\
36,93 \\
36,74 \\
36,78 \\
32,98\end{array}$ \\
\hline
\end{tabular}

\footnotetext{
* Abweichende Meßbedingungen: $20 \mathrm{~g} / \mathrm{l}$ Natriumchlorid im Test, $\mathrm{t}_{2}=10 \mathrm{~min}(\mathrm{NR}=10)$

** Kinetische Messung entsprechend Abbildung 11a

*** Messung gegen den Reagenzien-Leerwert entsprechend Abbildung 11b
} 


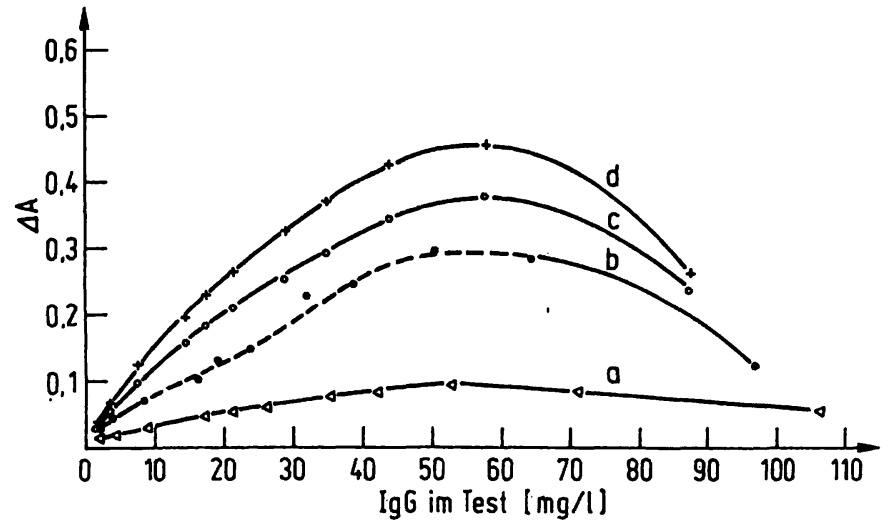

Abb. 10. Trübungskurven an parallel messenden Automaten bei gleicher Beschleunigung der Immunreaktion: (a) ABA100, (b) GEMSAEC, (c) CentrifiChem, (d) Cobas Bio. In dem gestrichelt gezeichneten Bereich ergeben sich Meßschwankungen. Reaktion smedium: $9 \mathrm{~g} / \mathrm{l} \mathrm{NaCl}$, $10 \mathrm{~g} /$ Polyethylenglykol 6000, Antiserum 1:14,6. Weitere Meßbedingungen siehe „Material und Methoden“.

in der Küvette zusammen etwa 15 Sekunden benötigt werden, ergibt sich ein Meßbeginn nach 15 und ein Meßintervall von maximal 45 Sekunden. Bei gleichem Reaktionsmedium wie in Abbildung 10 erhält man eine Trübungskurve, deren aufsteigender Ast einen Wendepunkt aufweist und als Folge der vergleichsweise kurzen Meßdauer etwas rechtsver schoben beginnt (Abb. 11a).

Durch Herabsetzung der Geschwindigkeit gelingt es zwar, einen Wendepunkt zu vermeiden, jedoch engt sich der potentielle Meßbereich durch Linksverschiebung des Maximums und weiter rechtsverschobenen Beginn der Trübungskurve ein. Zugleich erhöht sich die Gefahr, Antigenkonzentrationen im Bereich des abfallenden Astes der Trübungskurve zu erfassen. $\mathrm{Zu}$ berücksichtigen ist weiterhin, daß durch chargenbedingte Aviditäts- und/ oder Titerunterschiede der Antiseren eine weitere Linksverschiebung des Maximums erfolgen kann. Sollen kinetische fixed-time-Messungen durchgefüht werden, ist es daher sinnvöll, unregelmäßig ansteigende Referenzkur-

\section{Literatur}

1. Buffone, G. J., Savory, J. \& Cross, R. E. (1974), Clin. Chem. 20,1320-1323.

2. Baffone, G. J., Cross, R. E., Savory, J. \& Soodak, Ch. (1974), Anal. Chem. 46, 2047-2049.

3. Tiffaniy, T. O., Parella, J. M., Johnson, W. F. \& Burtis, C. A. (1974), Clin. Chem. 20, 1055-1061.

4. Buffone, G. J., Savory, J. \& Hermans, J. (1975), Clin. Chem. 21, 1735-1746.

5. Buffore, G. J., Savory, J., Cross, R. E. \& Hammond, J. E. (1975), Clin. Chem. 21, 1731-1734.

6. Sternberg, J. C. (1977), Clin. Chem. 23, 1456-1464.

7. Neumann, U., Kretzler, D., Munz, E., Schrappe, K.-H. \& Ziegenhorn, J. (1978), Lab. Med. 2, 62-68.

8. Ebeling, H. (1978), diese Z. 16, 191-195.

9. Malkus, H., Buschbaum, P. \& Castro, A. (1978), Clin. Chim. Acta 88, 523-530.

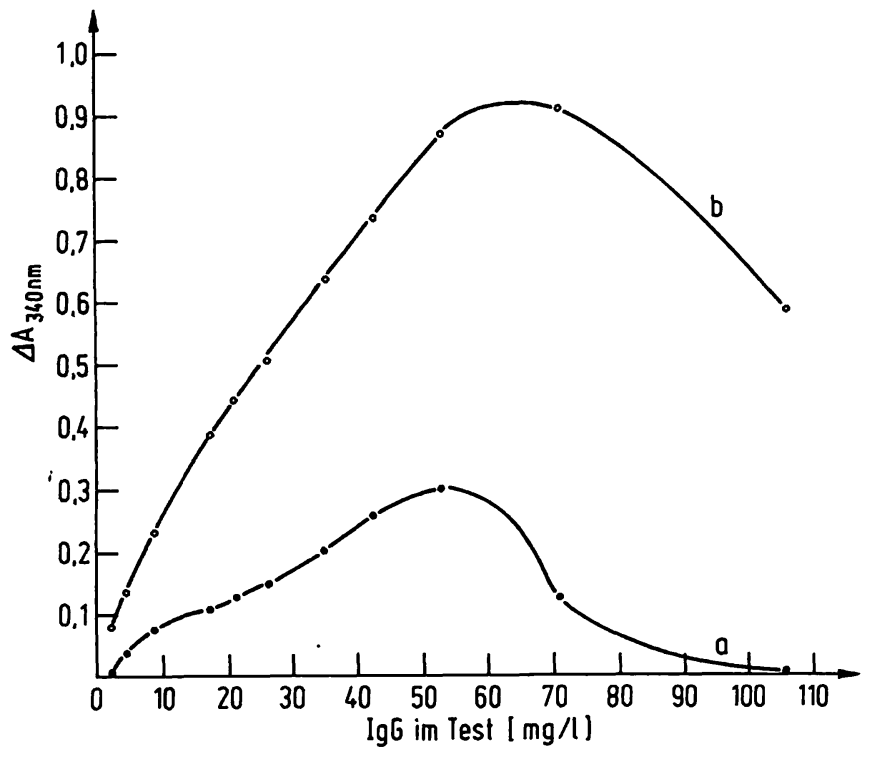

Abb. 11. Trübungskurven am Gilford 3500: (a) fixed-timeMessung mit $t_{1}=15 \mathrm{~s}$ und $t_{2}=45 \mathrm{~s}$, (b) Messung gegen den Reagenzien-Leerwert nach einer Reaktionsdauer von $15 \mathrm{~min}$. Reaktionsmedium: $9 \mathrm{~g} / \mathrm{l} \mathrm{NaCl}$, $10 \mathrm{~g} / 1$ Polyethylenglykol 6000, Antiserum 1:14,6. Messung bei $340 \mathrm{~nm}$.

ven wie in Abbildung 11a in Kauf zu nehmen und den Meßbereich auf den unteren, regelmäßig ansteigenden Bereich der Referenzkurve zu beschränken. Alternativ sind am Gilford 3500 Messungen gegen den ReagenzienLeerwert möglich. Kurve $11 \mathrm{~b}$ zeigt, daß sich bei gleichem Reaktionsmedium günstigere Trübungskurven ergeben, so daß das Antiserum besser ausgenutzt werden kann. Insgesamt werden an dem Gerät für Trübungsmessungen in einer Durchflußküvette mit kleinem Blendenquerschnitt erstaunlich gute Präzisionen erzielt (Tabelle 3).

\section{Danksagung}

Herrn H. Lodzik danken wir für die sorgfältige Mitarbeit bei experimentellen Untersuchungen.
10. Winkelmann, U. \& Storm, H. (1979), Z. Med. Labor Diagn. 20, 56-58.

11. van Oudheusden, A. P. M., van Buul, T. \& Luderer, T. K. J. (1979), Int. Lab. 29-32.

12. Finley, P. R., Williams, R. J., Lichti, D. A., Griffith, F. \& Thies, A. C. (1979), Clin. Chem. 25, 526-530.

13. Spencer, K. \& Prince, C. P. (1979), Clin. Chim. Acta 95, $263-276$.

14. Schultze, H. E. \& Schwick, G. (1959), Clin. Chim. Acta 4, $15-25$.

15. Hawkins, J. D. (1964), Immunology 7, 229-238.

16. Hawkins, J. D. (1965), Immunology 9, 107-117.

17. Marrack, J. R. \& Richards, C. B. (1971), Immunology 20, 1019-1040.

18. Killingsworth, L. M. \& Savory, J. (1973), Clin. Chem. 19, 403-407. 
19. Savory, J., Buffone, G. \& Reich, R. (1974), Clin. Chem. 20, 1071-1075.

20. Killingsworth, L. M., Buffone, G. J., Sonawane, M. B. \& Lunsford, G. C. (1974), Clin. Chem. 20, 1548-1552.

21. Lizana, J. \& Hellsing, K. (1974), Clin. Chem. 20, 11811186.

22. Killingsworth, L. M., Britain, C. E. \& Woodard, L. L. (1975), Clin. Chem. 21, 1465-1468.

23. Blom, M. \& Hjфrne, N. (1975), Clin. Chem. 21, 195-198.

24. Blom, M. \& Hjфrne, N. (1976), Clin. Chem. 22, 657-662.

25. Finley, P. R., Williams, R. J. \& Byers, J. M. (1976), Clin. Chem. 22, 1037-1041.
26. Van Munster, P. J. J., Hoelen, G. E. J. M., Samwel-Mantingh, M. \& Holtman-van Mèurs, M. (1977), Clin. Chim. Acta $76,377=388$.

27. Buffone, G. J., Lewis, S. A., Jolliff, C. R., Markowitz, H. \& Virella, G. (1979), Clin. Chēm. 25, 1009-1012.

28. Tiffany, T. O., Jansen, J. M., Burtis, C. A., Overton, J. B. \& Scott, D. (1972), Clin. Chem. 18, 829-840.

29. Creighton, W. D., Lambert, P. H. \& Miescher, P. A. (1973), J. Immunology $111,1219-1227$.

30. Peracino, A., Marcovina, S. \& Fenili, D. (1978), La Ricerca Clin. Lab. 8, 113-124.

31. Wider, G., Hotschek, H., Findeis, I. \& Bayer, P. M. (1979), Lab. Med. 3, 153-156.

Dr. R. Müller-Matthesius BEHRINGWERKE AG

Abteilung Reagenzien-Forschung D-3550 Marburg/Lahn 1 